Sonic Scope: New Approaches to Audiovisual Culture

\title{
Hugo Craft-Stanley - Beyond the Blue Horizon
}

\author{
Hugo Craft-Stanley
}

Published on: Feb 15, 2021

License: Creative Commons Attribution 4.0 International License (CC-BY 4.0). 


\section{Beyond the Blue Horizon}

Hugo Craft-Stanley, Goldsmiths, University of London

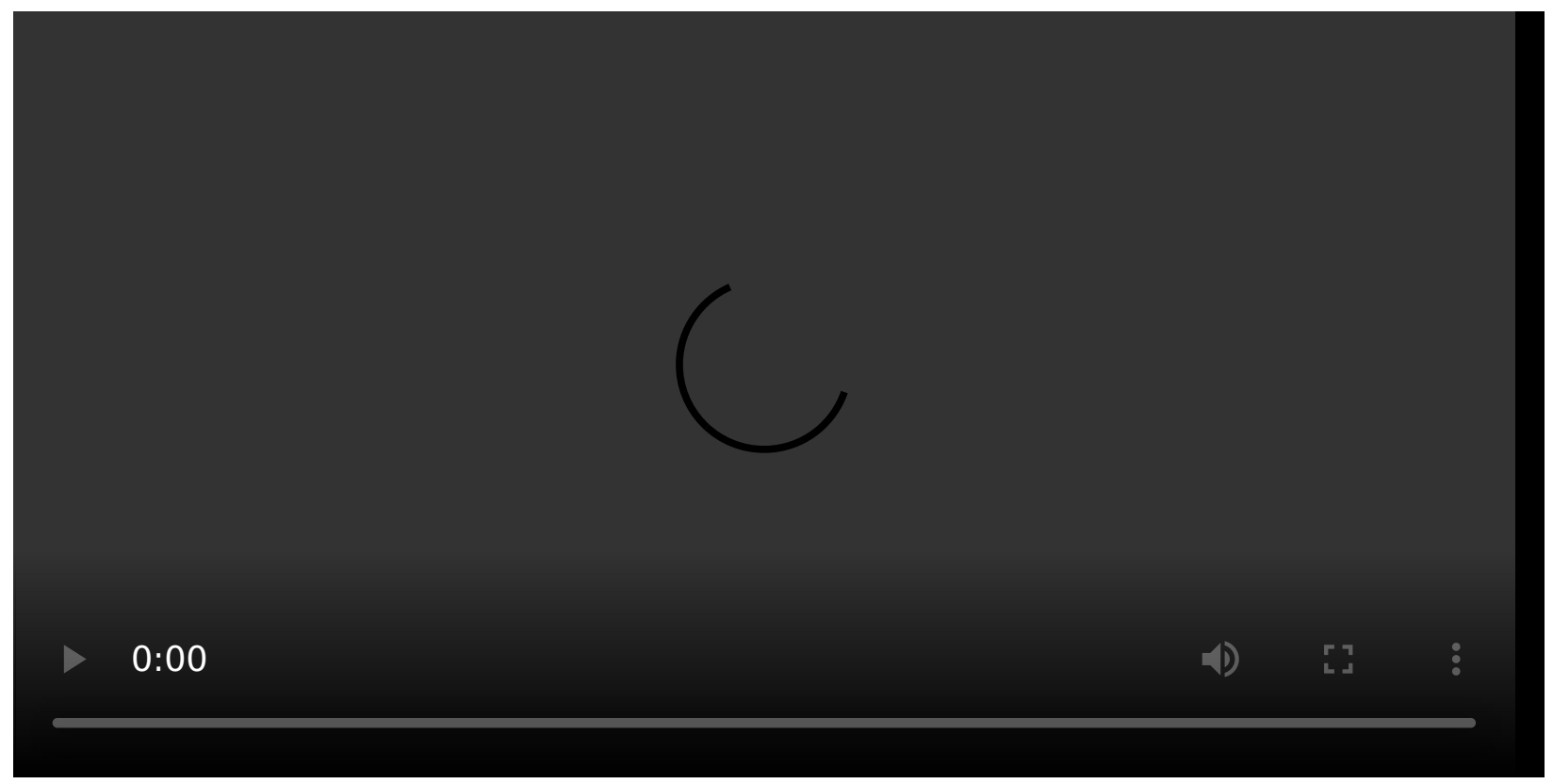

Beyond The Blue Horizon is a montage of found audiovisual materials structured into three soundimages.

These found materials include looped VHS playback glitches, sounds gleaned from an old Christmas mixtape found in my garage, an iPhone video of me playing the piano in 2018 , sections of an unknown film (appropriated from a VHS tape chosen at random in the university library) and fragments of schmaltzy tunes from a tape of love songs.

Stuttering and looped, these grains of the past add up to a montaged journey through three audiovisual environments which might be described as something like:

\section{0:00 - 00:34: the hazed landscape}

... a hazed landscape stuck on pause - shapes looming out of the foggy distance, as voices break through the wind, as ... 


\section{0:35 - 01:36 - the pianist in the mirror}

... as, behind the veil, the pianist in the mirror plays through and around time, a transmission conjuring ...

\section{1:37 - 02:14 - the clock at the end of time}

... conjuring the clock at the end of time, where we wait, and wait, by the racetrack. 